\title{
Anticipative and non-anticipative controller design for network control systems
}

\author{
Payam Naghshtabrizi ${ }^{1}$ and João P. Hespanha ${ }^{2}$ \\ 1 Department of Electrical and Computer Engineering, Univ. of California, Santa \\ Barbara, CA 93106-9560 payam@ece.ucsb.edu \\ 2 Department of Electrical and Computer Engineering, Univ. of California, Santa \\ Barbara, CA 93106-9560 hespanha@ece.ucsb.edu
}

Summary. We propose a numerical procedure to design a linear output-feedback controller for a remote linear plant in which the loop is closed through a network. The controller stabilizes the plant in the presence of delays, sampling, and packet dropouts in the (sensor) measurement and actuation channels. We consider two types of control units: anticipative and non-anticipative. In both cases the closedloop system with delays, sampling, and packet dropouts can be modeled as delay differential equations. Our method of designing the controller parameters is based on the Lyapunov-Krasovskii theorem and a linear cone complementarity algorithm. Numerical examples show that the proposed design method is significantly better than the existing ones.

\section{Introduction}

Network Control Systems (NCSs) are spatially distributed systems in which the communication between plants, sensors, actuators, and controllers occurs through a shared band-limited digital communication network. Using network as a medium to connect spatially distributed elements of the system results in flexible architectures and generally reduces wiring and maintenance cost, since there is no need for point to point wiring. Consequently, NCSs have been finding application in a broad range of areas such as mobile sensor networks, remote surgery, haptics collaboration over the Internet and unmanned aerial vehicles [7]. However, the use of a shared network, in contrast to using several dedicated independent connections, introduces new challenges.

To transmit a continuous-time signal over a network, the signal must be appropriately sampled to be carried over a network as atomic units called packet. Hence there are some similarities between NCSs and sampled-data systems due to the sampling effect. However, NCSs are significantly different from standard sampled-data systems since the delay in the feedback loop can be highly variable due to both access delay (i.e., the time it takes for a 
shared network to accept data) and transmission delay (i.e., the time during which data are in transit inside the network). Both types of delays depend on highly variable network conditions such as congestion and channel quality. Since access and transmission delays have the same effect with respect to the stability of NCSs, throughout the paper we use the term (NCS) delay to refer to access/transmission delay.

Another significant difference between NCSs and standard digital control is the possibility that data may be lost while in transit through the network. Typically, packet dropout results from transmission errors in physical network links (which is far more common in wireless than in wired networks) or from buffer overflows due to congestion. Long transmission delays sometimes result in out of order delivery, which can amount to a packet dropout if the receiver discards "outdated" arrivals. Reliable transmission protocols, such as TCP, guarantee the eventual delivery of packets. However, these protocols are not appropriate for NCSs since the re-transmission of old data is generally not very useful.

We consider two types of control units: non-anticipative and anticipative. A non-anticipative control unit sends control updates at times $t_{\ell}^{a}, \ell \in \mathbb{N}$, equal to $\left\{u_{\ell}: \forall \ell \in \mathbb{N}\right\}$, where $u_{\ell}$ is a single-value control command to be applied to the plant and held until the next control update arrives. An observerbased controller, constructs estimate $\hat{x}$ of the plant's state $x$. These estimates are used to compute the updates $u_{\ell}:=-K \hat{x}\left(t_{\ell}^{a}\right)$ sent through the actuation channel to the actuators at times $t_{\ell}^{a}, \ell \in \mathbb{N}$ suffering a (possibly variable) delay of $\tau_{\ell}^{a} \geq 0$. In a loss-less network, the control signal $u(t)$ is therefore updated according to

$$
u(t)=u_{\ell}=-K \hat{x}\left(t_{\ell}^{a}\right), \quad \forall t \in\left[t_{\ell}^{a}+\tau_{\ell}^{a}, t_{\ell+1}^{a}+\tau_{\ell+1}^{a}\right), \ell \in \mathbb{N} .
$$

An anticipative controller attempts to compensate the sampling and delay introduced by the actuation channel. For simplicity, we assume that the actuation channel is sampled with constant sampling interval $h^{a}=t_{\ell+1}^{a}-t_{\ell}^{a}$, $\forall \ell \in \mathbb{N}$ and that its delay is constant and equal to $\tau^{a}=\tau_{\ell}^{a}, \forall \ell \in \mathbb{N}$. At each sampling time $t_{\ell}^{a}=\ell h^{a}, \ell \in \mathbb{N}$ the controller sends a time-varying control signal $u_{\ell}(\cdot)$ that should be used from the time $\ell h^{a}+\tau^{a}$ at which it arrives until the time $(\ell+1) h^{a}+\tau^{a}$ at which the next control update will arrive. This leads to

$$
u(t)=u_{\ell}(t), \quad \forall t \in\left[\ell h^{a}+\tau^{a},(\ell+1) h^{a}+\tau^{a}\right), \ell \in \mathbb{N},
$$

where $u_{\ell}(t)$ is equal to $-K \hat{x}(t)$. However, the estimates $\hat{x}(\cdot)$ needed in the interval $\left[\ell h^{a}+\tau^{a},(\ell+1) h^{a}+\tau^{a}\right)$ must be available at the transmission time $\ell h^{a}$, which requires the control unit to estimate the plant's state up to $h^{a}+\tau^{a}$ time units into the future.

Branicky et al. [1], [15] model NCSs as discrete-time systems. However, this approach requires the assumption that the total delay in the control 
loop is smaller than the sampling interval, otherwise the analysis becomes significantly more complex [10]. Alternatively NCSs can be modeled as delay differential equations (DDEs) for which the after mentioned restriction can be easily lifted [7]. An NCS with LTI plant model, anticipative or non-anticipative controller, with delays, sampling, and packet dropouts can be modeled as a DDE of the form

$$
\dot{\bar{x}}(t)=A_{0} \bar{x}(t)+\sum_{i=1}^{2} A_{i} \bar{x}\left(t-\tau_{i}(t)\right), \tau_{i}(t) \in\left[\tau_{i \min }, \tau_{i \max }\right), \dot{\tau}_{i}(t)=1 \text { a.e. }
$$

The delay's bounds $\tau_{i \min }$ and $\tau_{i \max }$ for $i \in\{1,2\}$, are positive and depend on the sampling intervals, maximum number of consecutive packet dropouts, and upper and lower bounds on the delay in the measurement and the actuation channels. We find sufficient conditions for the stability of (1), based on a Lyapunov-Krasovskii functional, in the form of matrix inequalities. For a given controller, the matrix inequalities turn out to be linear (LMIs). However, in design problems in which the controller parameters also appear as unknowns, the matrix inequalities are bilinear (BMIs) and non-convex. We propose a numerical method based on the linear cone complementarity algorithm introduced in [6] to solve this problem. This method converts the feasibility of the original non-convex matrix inequalities into a sequence of the convex optimizations of a linear function subject to a set of LMIs, which can be effectively solved by numerical packages such as MATLAB.

\section{Related work}

The stability of NCSs has received significant attention in the literature. Zhang and Branicky [19] model NCSs with time-varying sampling intervals as hybrid systems and find sufficient condition for stability. Montestruque and Antsaklis [11] study the stability of model-based NCSs in which the network delays are negligible and the measurement channel is loss-less and the controller is directly connected to the plant. The authors use an explicit model of the plant to produce an estimate of the plant's state between transmission times which allows them to reduce the network usage. Lin et al. [9] investigate the stability of NCSs with uncertain time delays and packet dropouts in the framework of switched systems. Significant work has been devoted to finding upper bounds on transmission intervals, i.e., $t_{k+1}-t_{k}, \forall k \in \mathbb{N}$ for which stability of the closed-loop is guaranteed. These upper bounds are sometimes called the maximum allowable transfer interval (MATI). Walsh et al. [16, 17] find MATI for linear and nonlinear systems. Nesic and Teel [14] study the input-output stability properties of general nonlinear NCSs using an argument based on small gain theorem. Yu et al. [18] design an observer-type output feedback controller to stabilize a plant through a network with admissible bounds on data packet losses and delays, by modeling NCSs as DDEs and using the Razumikhin theorem to prove the stability of DDEs. Our work 
is inspired by [18], but instead of the Razumikhin theorem, which generally leads to conservative designs, our analysis is based on a new descriptor system approach and the Lyapunov-Krasovskii functional proposed by Fridman and Shaked [4]. We refer the readers to [7] for a more through review of related literature. The results in this paper generalized those in [13] by considering variable intervals.

\section{Notations}

The notation is introduced as and when necessary. The set of all non-negative integers is denoted by $\mathbb{N}$. Superscripts 's' and 'a' are used to refer to the (sensor) measurement and the actuator channels and subscripts $k$ and $j$ are discrete indices taking values in $\mathbb{N}$ used for the measurement and the actuator channel events respectively. For example $t_{k}^{s}$ and $t_{j}^{a}$ refer to the $k$-th and the $j$ th sampling times in the measurement and the actuation channels respectively.

This paper is organized as follows: In section 2 we introduce anticipative and non-anticipative control units. We show that the resulted closed-loop systems can be written as (1). In section 3, we provide a sufficient condition for asymptotic stability of (1) as a set of LMIs. In section 4 a numerical procedure is proposed to design a controller that stabilizes the plant for given admissible bound on delays, number of consecutive packet dropouts and sampling intervals. Then through examples we illustrate the use of our method.

\section{Network control systems modeling}

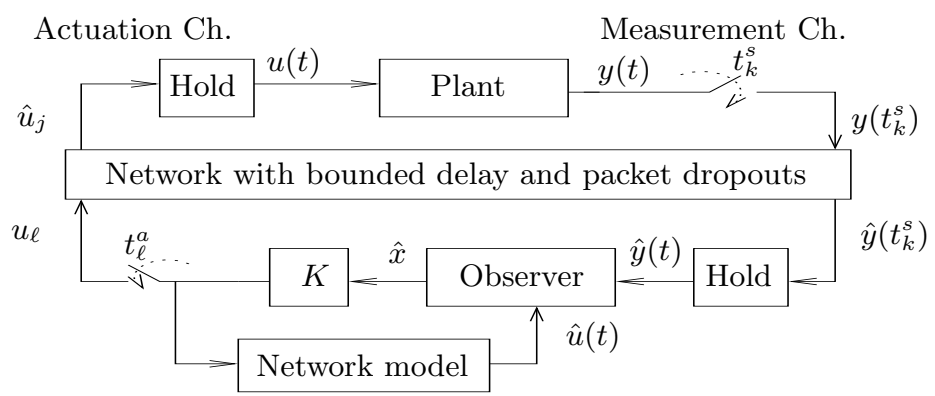

Fig. 1. Two-channel feedback NCS with observer-based controller.

Fig. 1 shows an NCS consisting of a plant, actuators, sensors, and a control unit where the plant, actuators, and sensors are compound. The plant is LTI with state space model of the form

$$
\dot{x}(t)=A x(t)+B u(t), \quad y(t)=C x(t),
$$


where $x(t) \in \mathbb{R}^{n}, u(t) \in \mathbb{R}^{l}, y(t) \in \mathbb{R}^{m}$ are the state, input, and output of the plant respectively. The measurements are sampled and sent at times $t_{k}^{s}, k \in \mathbb{N}$. Assuming, for now, that there are no dropouts, the measurements $\left\{y\left(t_{k}^{s}\right): k \in \mathbb{N}\right\}$ are received by the control unit at times $t_{k}^{s}+\tau_{k}^{s}$. These are used to construct an estimate of the plant state using

$$
\dot{\hat{x}}(t)=A \hat{x}(t)+B \hat{u}(t)+L\left(y\left(t_{k}^{s}\right)-C \hat{x}\left(t_{k}^{s}\right)\right), \quad \forall t \in\left[t_{k}^{s}+\tau_{k}^{s}, t_{k+1}^{s}+\tau_{k+1}^{s}\right),
$$

where $\hat{u}(t)$ is an estimate of the plant's input at time $t$. Since $u$ is constructed from data sent by the controller, in general we have $\hat{u}=u$. We consider two types of control units: non-anticipative and anticipative. The two units defer on the construction of the control signal $u$.

\subsection{Non-anticipative control unit}

Control signal The control unit sends control updates at times $t_{\ell}^{a}, \ell \in \mathbb{N}$, equal to $\left\{-K \hat{x}\left(t_{\ell}^{a}\right): \ell \in \mathbb{N}\right\}$. In the absence of drops, these arrive at the plant at times $t_{\ell+1}^{a}+\tau_{\ell+1}^{a}, \ell \in \mathbb{N}$, leading to

$$
u(t)=-K \hat{x}\left(t_{\ell}^{a}\right), \quad \forall t \in\left[t_{\ell}^{a}+\tau_{\ell}^{a}, t_{\ell+1}^{a}+\tau_{\ell+1}^{a}\right) .
$$

Delay differential equation formulation Defining

$$
\begin{array}{rlrl}
\tau^{s}(t) & :=t-t_{k}^{s}, & \forall t \in\left[t_{k}^{s}+\tau_{k}^{s}, t_{k+1}^{s}+\tau_{k+1}^{s}\right), \\
\tau^{a}(t):=t-t_{\ell}^{a}, & \forall t \in\left[t_{\ell}^{a}+\tau_{\ell}^{a}, t_{\ell+1}^{a}+\tau_{\ell+1}^{a}\right),
\end{array}
$$

which we call them fictitious delays (as opposed to the actual network delays $\tau_{k}^{s}$ and $\tau_{j}^{a}$ ), we can re-write (3) and (4) as ${ }^{3}$

$$
\begin{aligned}
& \dot{\hat{x}}(t)=A \hat{x}(t)+B \hat{u}(t)+L\left(y\left(t-\tau^{s}\right)-C x\left(t-\tau^{s}\right)\right), \\
& u(t)=-K \hat{x}\left(t-\tau^{a}\right) .
\end{aligned}
$$

Note that

$$
\begin{array}{lll}
\tau^{s} \in\left[\min _{k}\left\{\tau_{k}^{s}\right\}, \max _{k}\left\{t_{k+1}^{s}-t_{k}^{s}+\tau_{k+1}^{s}\right\}\right), & \forall k \in \mathbb{N}, \dot{\tau}^{s}=1 & \text { a.e. }, \\
\tau^{a} \in\left[\min _{\ell}\left\{\tau_{\ell}^{a}\right\}, \max _{\ell}\left\{t_{\ell+1}^{a}-t_{\ell}^{a}+\tau_{\ell+1}^{a}\right\}\right), & \forall k \in \mathbb{N}, \dot{\tau}^{a}=1 & \text { a.e. },
\end{array}
$$

Fig. 2.a shows the evolution of $\tau^{s}$ with respect to time where $\tau_{k}^{s}=\bar{\tau}^{s}, \forall k \in \mathbb{N}$ and constant sampling interval $t_{k+1}^{s}-t_{k}^{s}=h^{s}, \forall k \in \mathbb{N}$. The derivative of $\tau^{s}$ is almost always one, except at the times that new packets arrive to the controller side at $t_{k+1}^{s}+\bar{\tau}^{s}$, where $\tau^{s}$ (the fictitious delay) drops to $\bar{\tau}^{s}$ (the actual network delay).

\footnotetext{
${ }^{3}$ For simplicity of notation through the paper we use $\tau^{s}$ and $\tau^{a}$ as $\tau^{s}(t)$ and $\tau^{a}(t)$ respectively.
} 


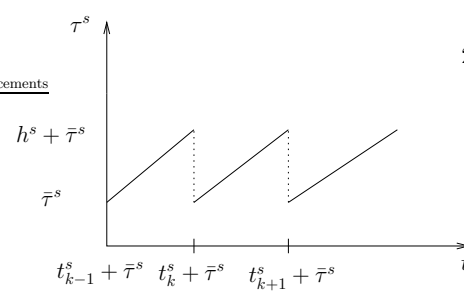

(a)

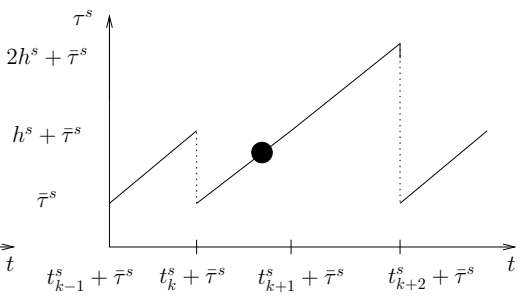

(b)

Fig. 2. Evolution of $\tau^{s}$ with respect to time when (a) There is no packet dropout, (b) The packet sent at $t_{k}^{s}$ is dropped.

Packet dropouts Packet dropouts in the measurement and actuation channels can be viewed as the delay that grow beyond the defined bounds. If $\mathrm{m}^{\mathrm{s}}$ and $m^{a}$ consecutive packet dropouts happen in the measurement and actuation channels then

$$
\begin{aligned}
& \tau^{s} \in\left[\min _{k}\left\{\tau_{k}^{s}\right\}, \max _{k}\left\{t_{k+m^{s}+1}^{s}-t_{k}^{s}+\tau_{k+m^{s}+1}^{s}\right\}\right), \\
& \tau^{a} \in\left[\min _{\ell}\left\{\tau_{\ell}^{a}\right\}, \max _{\ell}\left\{t_{\ell+m^{a}+1}^{a}-t_{\ell}^{a}+\tau_{\ell+m^{a}+1}^{a}\right\}\right) .
\end{aligned}
$$

Fig. 2.b shows the situation that the measurement packet sent at $t=t_{k}^{s}$ is dropped and as a result $\tau^{s}$ grows up to $2 h^{s}+\tau^{s}$.

Closed-loop Defining $e:=x-\hat{x}$, with regard to (2) and (5), the closed-loop can be written as

$$
\left[\begin{array}{l}
\dot{x}(t) \\
\dot{e}(t)
\end{array}\right]=\left[\begin{array}{cc}
A & 0 \\
0 & A
\end{array}\right]\left[\begin{array}{l}
x(t) \\
e(t)
\end{array}\right]+\left[\begin{array}{cc}
0 & 0 \\
0 & -L C
\end{array}\right]\left[\begin{array}{l}
x\left(t-\tau^{s}\right) \\
e\left(t-\tau^{s}\right)
\end{array}\right]+\left[\begin{array}{cc}
-B K & B K \\
0 & 0
\end{array}\right]\left[\begin{array}{l}
x\left(t-\tau^{a}\right) \\
e\left(t-\tau^{a}\right)
\end{array}\right]
$$

or alternatively, as

$$
\left[\begin{array}{c}
\dot{\hat{x}}(t) \\
\dot{e}(t)
\end{array}\right]=\left[\begin{array}{cc}
A & 0 \\
0 & A
\end{array}\right]\left[\begin{array}{l}
\hat{x}(t) \\
e(t)
\end{array}\right]+\left[\begin{array}{cc}
0 & L C \\
0 & -L C
\end{array}\right]\left[\begin{array}{l}
\hat{x}\left(t-\tau^{s}\right) \\
e\left(t-\tau^{s}\right)
\end{array}\right]+\left[\begin{array}{cc}
-B K & 0 \\
0 & 0
\end{array}\right]\left[\begin{array}{l}
\hat{x}\left(t-\tau^{a}\right) \\
e\left(t-\tau^{a}\right)
\end{array}\right] .
$$

\subsection{Anticipative control unit}

Control signal For simplicity, we assume that the actuation channel is sampled with constant sampling interval $h^{a}=t_{\ell+1}^{a}-t_{\ell}^{a}, \forall \ell \in \mathbb{N}$ and that its delay is constant and equal to $\bar{\tau}^{a}=\tau_{\ell}^{a}, \forall \ell \in \mathbb{N}$. At each sampling time $t_{\ell}^{a}=\ell h^{a}, \ell \in \mathbb{N}$ the controller sends a time-varying control signal $u_{\ell}(\cdot)$ that should be used from the time $\ell h^{a}+\bar{\tau}^{a}$ at which it arrives until the time $(\ell+1) h^{a}+\bar{\tau}^{a}$ at which the next control update will arrive. This leads to

$$
u(t)=u_{\ell}(t), \quad \forall t \in\left[\ell h^{a}+\bar{\tau}^{a},(\ell+1) h^{a}+\bar{\tau}^{a}\right), \ell \in \mathbb{N} .
$$

To stabilize $(2), u_{\ell}(t)$ should be equal to $-K \hat{x}(t)$, where $\hat{x}(t)$ is an estimate of $x(t)$. However, the estimates $\hat{x}(\cdot)$ needed in the interval $\left[\ell h^{a}+\bar{\tau}^{a},(\ell+1) h^{a}+\bar{\tau}^{a}\right)$ must be available at the transmission time $\ell h^{a}$, which requires the control unit to estimate the plant's state up to $h^{a}+\bar{\tau}^{a}$ time units into the future. 
Remark 1. Anticipative controllers send actuation signals to be used during time intervals of duration $h^{a}$, therefore the sample and hold blocks in Fig. 1 should be understood in a broad sense. In practice, the sample block would send over the network some parametric form of the control signal $u_{\ell}(\cdot)$ (e.g., the coefficients of a polynomial approximation to this signal).

State predictor An estimate $z(t)$ of $x\left(t+h^{a}+\bar{\tau}^{a}\right)$ is constructed as follows:

$$
\dot{z}(t)=A z(t)+B \hat{u}\left(t+h^{a}+\bar{\tau}^{a}\right)+L\left(\hat{y}\left(t_{k}^{s}\right)-C z\left(t_{k}^{s}-h^{a}-\bar{\tau}^{a}\right)\right),
$$

for $\forall t \in\left[t_{k}^{s}+\tau_{k}^{s}, t_{k+1}^{s}+\tau_{k+1}^{s}\right), k \in \mathbb{N}$. To compensate for the time varying delay and packet dropouts in the actuation channel, $z$ would have to estimate $x$ further into the future. Hence the assumptions of constant delay and loss-less actuation channel can be relaxed by predicting $x$ further into the future.

Control signal construction With such estimate available, the signal $u_{\ell}(t)$ sent at times $t_{\ell}^{a}=\ell h^{a}$, to be used in $\left[\ell h^{a}+\bar{\tau}^{a},(\ell+1) h^{a}+\bar{\tau}^{a}\right)$, is then given by

$$
u_{\ell}(t)=-K z\left(t-h^{a}-\bar{\tau}^{a}\right), \quad \forall t \in\left[\ell h^{a}+\bar{\tau}^{a},(\ell+1) h^{a}+\bar{\tau}^{a}\right), \ell \in \mathbb{N},
$$

which only requires the knowledge of $z($.$) in the interval t \in\left[(\ell-1) h^{a}, \ell h^{a}\right)$, and therefore is available at the transmission time $\ell h^{a}$.

Delay differential equation formulation Defining

$$
\tau^{s}(t):=t-t_{k}^{s}, \quad \forall t \in\left[t_{k}^{s}+\tau_{k}^{s}, t_{k+1}^{s}+\tau_{k+1}^{s}\right),
$$

and assuming that $\hat{u}=u$, we conclude from (6),(7), and (8) that

$$
\begin{aligned}
\dot{z}(t) & =(A-B K) z(t)+L\left(y\left(t-\tau^{s}\right)-C z\left(t-h^{a}-\bar{\tau}^{a}-\tau^{s}\right)\right), \\
\tau^{s}(t) & \in\left[\min _{k}\left\{\tau_{k}^{s}\right\}, \max _{k}\left\{t_{k+1}^{s}-t_{k}^{s}+\tau_{k+1}^{s}\right\}\right), \quad \forall k \in \mathbb{N}, \quad \dot{\tau}^{s}=1 \quad \text { a.e. }
\end{aligned}
$$

Closed-loop Defining $e(t):=x\left(t+h^{a}+\bar{\tau}^{a}\right)-z(t)$ and considering (2),(8), and (9), the closed-loop can be written as

$$
\left[\begin{array}{c}
\dot{z}(t) \\
\dot{e}(t)
\end{array}\right]=\left[\begin{array}{cc}
A-B K & 0 \\
0 & A
\end{array}\right]\left[\begin{array}{l}
z(t) \\
e(t)
\end{array}\right]+\left[\begin{array}{cc}
0 & L C \\
0 & -L C
\end{array}\right]\left[\begin{array}{c}
z\left(t-h^{a}-\bar{\tau}^{a}-\tau^{s}\right) \\
e\left(t-h^{a}-\bar{\tau}^{a}-\tau^{s}\right)
\end{array}\right] .
$$

\section{Stability of delay differential equations}

It was shown in section 2 that both types of control units result in the closedloop models of the following form

$$
\dot{\bar{x}}(t)=A_{0} \bar{x}(t)+\sum_{i=1}^{2} A_{i} \bar{x}\left(t-\tau_{i}(t)\right), \tau_{i}(t) \in\left[\tau_{i \min }, \tau_{i \max }\right), \dot{\tau}_{i}(t)=1 \text { a.e. }
$$


Until recently it was believed that the only available tool to study the stability of delay equations of the form (11) was the Razumikhin theorem. Fridman and Shaked [5] were able to use the Lyapunov-Krasovskii theorem to study the stability of system (11). In [3] they studied the stability of sampled-data systems with input delays as DDEs of the form (11) where $\tau_{1} \in\left[0, h^{s}\right)$. In sampled-data systems, at each sampling time delay drops to zero. However in NCSs as new information arrives the fictitious delay drops to the actual network delay. The next theorem gives a sufficient condition for the asymptotic stability of (11) where the matrices $A_{i}$ is $2 n \times 2 n$ for $i \in\{0,1,2\}$.

Theorem 1. The system (11) is asymptotically stable, if there exist $2 n \times 2 n$ matrices $P_{1}>0, P_{2}, P_{3}, S_{i}, R_{i}>0$ and $4 n \times 4 n$ matrices $Z_{1 i}, Z_{2 i}$ and $2 n \times 4 n$ matrices $T_{i}, i=1,2$, that satisfy the following set of matrix inequalities ${ }^{4}$ :

$$
\begin{aligned}
& {\left[\begin{array}{ccc}
\Psi P^{\prime} & {\left[\begin{array}{c}
0 \\
A_{1}
\end{array}\right]-T_{1}^{\prime} P^{\prime}\left[\begin{array}{c}
0 \\
A_{2}
\end{array}\right]-T_{2}^{\prime}} \\
* & -S_{1} & 0 \\
* & * & -S_{2}
\end{array}\right]<0,}
\end{aligned}
$$

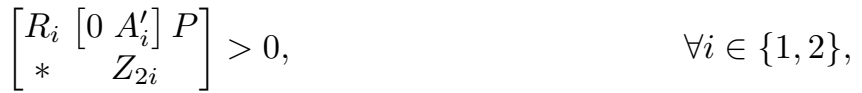

$$
\begin{aligned}
& {\left[\begin{array}{cc}
R_{i} & T_{i} \\
* & Z_{1 i}
\end{array}\right]>0, \quad \forall i \in\{1,2\},}
\end{aligned}
$$

where

$$
\begin{gathered}
P:=\left[\begin{array}{cc}
P_{1} & 0 \\
P_{2} & P_{3}
\end{array}\right], \quad \Psi:=P^{\prime}\left[\begin{array}{cc}
0 & I \\
A_{0} & -I
\end{array}\right]+\left[\begin{array}{cc}
0 & I \\
A_{0} & -I
\end{array}\right]^{\prime} P+\Phi \\
\Phi:=\sum_{i=1}^{2}\left(\left[\begin{array}{cc}
S_{i} & 0 \\
0 & \tau_{i \max } R_{i}
\end{array}\right]+\tau_{i \min } Z_{1 i}+\left(\tau_{i \max }-\tau_{i \min }\right) Z_{2 i}+\left[\begin{array}{c}
T_{i} \\
0
\end{array}\right]+\left[\begin{array}{c}
T_{i} \\
0
\end{array}\right]\right) .
\end{gathered}
$$

Proof of Theorem 1 See Appendix.

Remark 2. The "triangular" structure of (10) is unique to the anticipate controller. With this type of controller, if we choose $K$ so that $A-B K$ is Hurwitz, asymptotic stability of the NCS is equivalent to the asymptotic stability of the (decoupled) dynamics of the error $e(t):=x\left(t+h^{a}+\bar{\tau}^{a}\right)-z(t)$, which is given by the following DDE

$$
\dot{e}(t)=A e(t)-L C e(t-\tau(t)), \quad t \geq 0,
$$

with $\tau \in\left[\tau_{\min }, \tau_{\max }\right), \dot{\tau}=1$, a.e., where

$\tau_{\min }:=\min _{k \in \mathbb{N}}\left\{\tau_{k}^{s}+h^{a}+\bar{\tau}^{a}\right\}, \tau_{\max }:=\max _{k \in \mathbb{N}}\left\{t_{k+1+m^{s}}^{s}-t_{k}^{s}+\tau_{k+1+m^{s}}^{s}+h^{a}+\bar{\tau}^{a}\right\}$,

and Theorem 1 provides the stability test with $A_{0}=A$ and $A_{1}=-L C$.

\footnotetext{
${ }^{4}$ Matrix entries denoted by ${ }^{\prime} *$, are implicitly defined by the fact that the matrix is symmetric.
} 
Remark 3. Suppose that the matrices of the system $\Omega:=\left[\begin{array}{lll}A_{0} & A_{1} & A_{2}\end{array}\right]$ are not exactly known and that instead they are given as polytopic uncertainty given by

$$
\Omega \in\left\{\sum_{j=1}^{N} f_{j} \Omega_{j}, \quad 0 \leq f_{j} \leq 1, \quad \sum_{j=1}^{N} f_{j}=1\right\},
$$

where the $N$ vertices of the polytope are described by $\Omega_{j}:=\left[\begin{array}{lll}A_{0}^{j} & A_{1}^{j} & A_{2}^{j}\end{array}\right]$. Stability of the system can be checked by solving the LMIs in Theorem 1 for each of the individual vertices and the same matrix variables.

\section{Observer-based controller design for NCSs}

When the controller parameters $L$ and $K$ are known, the system matrices $A_{i}$ for $i \in\{0,1,2\}$, are constant and known. Hence (12a)-(12c) are in the form of LMIs and the stability of NCSs can be established easily. However when $L$ and $K$ are also variables to be defined, since $A_{i}$ contain unknowns, the matrix inequalities in Theorem 1 are in the form of BMIs. The next lemma taken from [2], plays a central role in transforming BMIs to a suitable form for a numerical algorithm we will develop.

Lemma 1. Assume that $Q$ is a symmetric matrix. There exists a symmetric matrix $N>0$ such that

$$
J^{\prime} N U+U^{\prime} N J+Q<0,
$$

if and only if there exist symmetric matrices $X, Y$ and a scalar $\alpha>0$ such that $X=\alpha^{2} Y^{-1}$ and

$$
\left[\begin{array}{cc}
U^{\prime} X U & -Q \\
* & J^{\prime}+\alpha U^{\prime}
\end{array}\right]>0 .
$$

The proof is obtained by Schur Lemma and the equation $X=\alpha N$. Lemma 1 changes the matrix inequality (14), with products of unknowns $J$ and $N$, into the inequality (15) in which such products are absent. However, (15) requires the non-convex constraint $X=\alpha^{2} Y^{-1}$. Our goal now is to rewrite the matrix inequalities in Theorem 1 in the form of (14) and ultimately in the form of (15). It turns out that this form is suitable for developing a numerical procedure to compute $K, L$, and the other matrix variables in Theorem 1. Suppose that $P_{2}>0, P_{3}>0$, after matrix manipulations (12a) can be written as $J_{0}(K, L)^{\prime} N U_{0}+U_{0}^{\prime} N J_{0}(K, L)+Q_{0}<0$, where

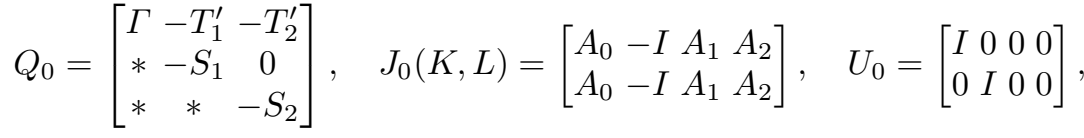

$$
\begin{aligned}
& N=\left[\begin{array}{cc}
P_{2} & 0 \\
0 & P_{3}
\end{array}\right]>0, \quad \Gamma=\left[\begin{array}{cc}
0 & P_{1} \\
P_{1} & 0
\end{array}\right]+\Phi .
\end{aligned}
$$


The matrix inequality (12b) can be written as $J_{i}(K, L)^{\prime} N U_{i}+U_{i}^{\prime} N J_{i}(K, L)+$ $Q_{i}<0$, where

$$
Q_{i}=-\left[\begin{array}{cc}
R_{i} & 0 \\
0 & Z_{2 i}
\end{array}\right], \quad J_{i}(K, L)=-\left[\begin{array}{ccc}
A_{i} & 0 & 0 \\
A_{i} & 0 & 0
\end{array}\right], \quad U_{i}=\left[\begin{array}{ccc}
0 & I & 0 \\
0 & 0 & I
\end{array}\right],
$$

for $i \in\{1,2\}$. Theorem 1 can be read as the following theorem:

Theorem 2. The anticipative or non-anticipative controller given in section 2 with the matrix variables $K$ and $L$ asymptotically stabilizes the plant (2) for given $\tau_{i \min }$ and $\tau_{i \max }$, if there exist $2 n \times 2 n$ matrices $P_{1}>0, X_{1}>0, X_{2}>0$, $Y_{1}, Y_{2}, S_{i}, R_{i}>0,4 n \times 4 n Z_{1 i}, Z_{2 i}, 2 n \times 4 n$ matrices $T_{i}, n \times 1$ matrix $L$, $1 \times n$ matrix $K$ and $\alpha>0$ that satisfy the following matrix inequalities for $i \in\{1,2\}$ :

$$
\left[\begin{array}{cc}
U_{0}^{\prime} X U_{0}-Q_{0} & J_{0}^{\prime}+\alpha U_{0}^{\prime} \\
* & Y
\end{array}\right]>0,\left[\begin{array}{cc}
U_{i}^{\prime} X U_{i}-Q_{i} & J_{i}^{\prime}+\alpha U_{i}^{\prime} \\
* & Y
\end{array}\right]>0,\left[\begin{array}{cc}
R_{i} & T_{i} \\
* & Z_{1 i}
\end{array}\right]>0
$$

where

$$
X=\alpha^{2} Y^{-1}, \quad X=\left[\begin{array}{cc}
X_{1} & 0 \\
0 & X_{2}
\end{array}\right], \quad Y=\left[\begin{array}{cc}
Y_{1} & 0 \\
0 & Y_{2}
\end{array}\right] .
$$

Since $A_{0}, A_{1}, A_{2}$ are linear functions of $K$ and $L$, the matrix inequalities in (16) are in the form of LMIs also in the unknowns. However, the condition $X=\alpha^{2} Y^{-1}$ is not convex. In the next section we introduce a numerical procedure to solve this non-convex problem.

Remark 4. A simple but conservative way to make the matrix inequalities in Theorem 1 suitable for controller synthesis for anticipative controllers with regard to Remark 2, consists of requiring that $P_{2}>0, P_{3}=\rho P_{2}$, for some positive constant $\rho>0$ and making the (bijective) change of variables $Y=P_{2} L$, which transforms (12) into

$$
\left[\begin{array}{cc}
\Psi & -\left[\begin{array}{c}
Y C \\
\rho Y C
\end{array}\right]-T^{\prime} \\
* & -S
\end{array}\right]<0, \quad\left[\begin{array}{cc}
R & -\left[C^{\prime} Y^{\prime} \rho C^{\prime} Y^{\prime}\right] \\
* & Z_{2}
\end{array}\right]>0, \quad\left[\begin{array}{cc}
R & T \\
* & Z_{1}
\end{array}\right]>0,
$$

with $\Psi$ given by (13). This inequality is linear in the unknowns and can therefore be solved using efficient numerical algorithms. The observer gain is found using $L=P_{2}^{-1} Y$. This procedure introduces some conservativeness because it restricts $P_{3}$ to be a scalar multiple of $P_{2}$.

\section{Numerical procedure}

We modify the complementarity linearization algorithm introduced in [6] to construct a procedure to determine the feasibility of the matrix inequalities in Theorem 2 by solving a sequence of LMI's as follows: 
1. Pick $\alpha$.

2. Find a feasible solution denoted by $X_{0}, Y_{0}$ for the set of LMIs (16) and

$$
\left[\begin{array}{cc}
X & I \\
I & \alpha^{-2} Y
\end{array}\right] \geq 0
$$

3. Find the feasible solution denoted by $X_{j+1}, Y_{j+1}$ that solves the following problem

$$
\begin{array}{r}
\Sigma_{j}: \min \operatorname{tr}\left(X_{j} Y+X Y_{j}\right) \\
\text { subject to }(16),(17) .
\end{array}
$$

4. We choose satisfying (12a) and (12b) with $N=\alpha^{-1} X$ as the stopping criterion. If the matrix inequalities are satisfied, exit. Otherwise set $j=$ $j+1$ and go to step 3 if $j<c$ (a preset number).

The sequence $\Sigma_{j}$ is monotonically decreasing, lower bounded by $8 n \times \alpha^{-2}$ achieved at $X=\alpha^{2} Y^{-1}$, if the LMIs in (16) are feasible with the constraint $X=\alpha^{2} Y^{-1}$.

It is numerically very difficult to obtain the minimum such that the $\operatorname{tr}\left(X_{j} Y_{j+1}+\right.$ $X_{j+1} Y_{j}$ ) equals to $8 n \times \alpha^{-2}$. Instead we choose $(12 \mathrm{a})$ and $(12 \mathrm{~b})$ with $N=$ $\alpha^{-1} X$ as the stopping criterion. If after c times the stopping criterion is not satisfied we pick another $\alpha$ and we continue line search until the stopping criteria is satisfied.

\section{Examples}

Example 1. [1, 19] Consider the following plant

$$
\left[\begin{array}{c}
\dot{x}_{1} \\
\dot{x}_{2}
\end{array}\right]=\left[\begin{array}{cc}
0 & 1 \\
0 & -0.1
\end{array}\right]\left[\begin{array}{l}
x_{1} \\
x_{2}
\end{array}\right]+\left[\begin{array}{c}
0 \\
0.1
\end{array}\right] u,
$$

and assume that all the states are available for feedback. We assume there is no delay and packet dropout in the network and the state feedback $K=$ [3.75 11.5] stabilizes the plant. Branicky et al. [1] model the system as a hybrid system and show that the closed-loop is stable as long as the sampling intervals are constant and equal to $4.5 \times 10^{-4}$. Later Zhang and Branicky [19] find the less conservative upper bound 0.0593 for variable sampling intervals. Based on an exhaustive search the same authors find that the "true" upper bound is roughly 1.7194 . The maximum variable sampling interval based on Theorem 1 is 0.8965 .

Suppose now that just the first state is available for the feedback. Based on Theorem 2 and the proposed algorithm, we design a controller to stabilize the plant when the sampling interval of the measurement channel is constant and equal to $h_{k}^{s}=0.5, \forall k \in \mathbb{N}$. A non-anticipative control unit with

$$
K=\left[\begin{array}{ll}
3.3348 & 9.9103
\end{array}\right], \quad L=\left[\begin{array}{ll}
0.6772 & 0.1875
\end{array}\right]^{\prime},
$$




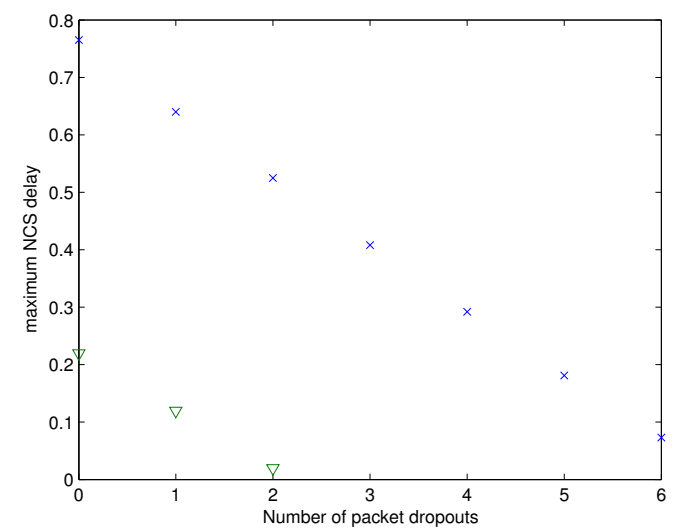

Fig. 3. Number of consecutive packet dropouts versus the maximum of actual network delay. Results in our paper and [18] are shown by $*$ and $\nabla$ respectively.

stabilizes the plant for any actuation sampling intervals such that $h_{\ell}^{a} \leq$ $0.7330, \forall \ell \in \mathbb{N}$. With the same measurement sampling interval, an anticipative control unit with

$$
K=\left[\begin{array}{ll}
28.5347 & 83.8626
\end{array}\right], \quad L=\left[\begin{array}{ll}
0.3518 & 0.0492
\end{array}\right]^{\prime},
$$

stabilizes the plant for any actuation sampling intervals such that $h_{\ell}^{a} \leq$ $0.976, \forall \ell \in \mathbb{N}$. As we expected the anticipative controller stabilizes the plant for larger sampling intervals.

Example 2. Yu et al. [18] consider the following state space plant model

$$
\left[\begin{array}{c}
\dot{x}_{1} \\
\dot{x}_{2}
\end{array}\right]=\left[\begin{array}{cc}
-1.7 & 3.8 \\
-1 & 1.8
\end{array}\right]\left[\begin{array}{l}
x_{1} \\
x_{2}
\end{array}\right]+\left[\begin{array}{c}
5 \\
2.01
\end{array}\right] u, \quad y(t)=[10.14 .5]\left[\begin{array}{l}
x_{1} \\
x_{2}
\end{array}\right]
$$

with the controller directly connected to the actuator, which means that there is no delay, sampling, and packet dropouts in the actuation channel. Yu et al. [18] show that the controller with gains

$$
K=\left[\begin{array}{ll}
-.2115 & 2.5044
\end{array}\right], \quad L=\left[\begin{array}{ll}
0.1043 & 0.0518
\end{array}\right]^{\prime},
$$

stabilizes the system for any fictitious delay $\tau^{s}$ in the interval $[0,0.3195]$. Consequently as long as $\left(t_{k+1+m^{s}}-t_{k}\right)+\tau_{k}^{s} \leq 0.3195$ holds, the closed-loop system remains stable. Fig. 3 shows the number of consecutive packet dropouts versus the actual network delay such that the system remains stable in which $h^{s}:=t_{k+1}-t_{k}=.1 s, \forall k \in \mathbb{N}$. Our result reveals a significant improvement in comparison to [18]. For instance, when the measurement channel is lossless $\left(m^{s}=0, \tau_{i \max }-\tau_{i \min }=h^{s}\right)$, the LMIs in Theorem 2 are feasible up to $\tau_{i \max }=0.865$ which means the closed-loop system with 


$$
K=\left[\begin{array}{ll}
-1.7436 & 1.1409
\end{array}\right], \quad L=\left[\begin{array}{ll}
0.0675 & 0.0267
\end{array}\right]^{\prime},
$$

is stable for any $\tau_{k}^{s} \in[0,0.765], \forall k \in \mathbb{N}$. If we assume a maximum number of consecutive packet dropouts $m^{s}=6$ (which corresponds to $\tau_{i \max }-\tau_{i \min }=$ $\left.7 \times h^{s}\right)$, the LMIs are feasible up to $\tau_{i \max }=0.773$ and the closed-loop system with

$$
K=\left[\begin{array}{ll}
-0.5310 & 0.1668
\end{array}\right], \quad L=\left[\begin{array}{ll}
0.0564 & 0.0221
\end{array}\right]^{\prime},
$$

is stable for any $\tau_{k}^{s} \in[0,0.073], \forall k \in \mathbb{N}$. As expected, for a smaller number of consecutive packet dropouts, the system remains stable for larger $\tau_{i \text { max }}$.

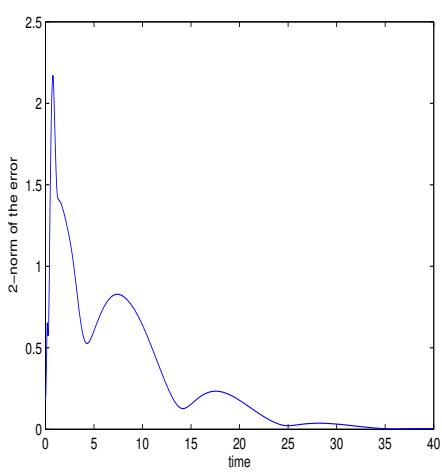

(a)

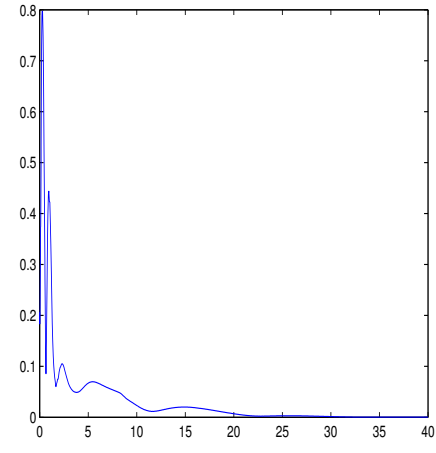

(b)

Fig. 4. Simulation results considering the nonlinear model for the inverted pendu$\operatorname{lum}$ (a) $L_{2}$ norm of state $x$, (b) $L_{2}$ norm of error $e=x\left(t+h^{a}\right)-z(t)$.

Example 3. [8] In this example we design an anticipative controller for an inverted pendulum. The linearized model is given by

$$
\dot{x}=\left[\begin{array}{cccc}
0 & 0 & 1 & 0 \\
0 & 0 & 0 & 0 \\
1 & 0 & -3 \frac{m g}{m+4 M} & -4 \frac{\beta+\epsilon}{m+4 M} \\
0 & 3 \frac{(M+m) g}{l(m+4 M)} & 3 \frac{\beta+\epsilon}{l(m+4 M)} & 0
\end{array}\right] x+\left[\begin{array}{c}
0 \\
0 \\
\frac{4 \alpha}{m+4 M} \\
\frac{-3 \alpha}{l(m+4 M)}
\end{array}\right] u,
$$

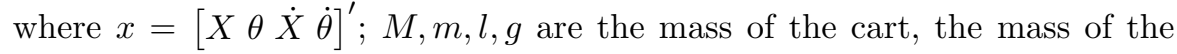
pendulum, half of the length of the pendulum, acceleration due to the gravity, $\alpha, \beta$ are motor specifications and $\epsilon$ is the viscous friction. The value of 
variables are given in [8], and $\epsilon=2$. The sampling interval of the sensor and measurement channels are equal to 0.05 , and we assume $\tau_{\ell}^{a}=0, \forall \ell \in \mathbb{N}$. Our goal is to find $K$ and $L$ such that the upper bound on the tolerable network delays in the measurement channel $\tau_{k}^{s}$ is maximized. The LMIs are feasible up to $\tau_{\text {imax }}=0.195$ when $\tau_{i \min }=0.05$ (regarding $(10), \tau_{i \min }=h^{a}+\bar{\tau}^{a}$ where we assumed $\bar{\tau}^{a}=0$ ). The observer gain $L$ is obtained by solving the LMIs in Remark 4. The state feedback gain $K$ is chosen such that $A-B K$ is asymptotically stable. With

$$
\begin{aligned}
L & =\left[\begin{array}{cccc}
0.9706 & -0.2082 & 0.0369 & 0.4441 \\
-0.1100 & -0.7815 & 5.1597 & 26.4242
\end{array}\right]^{\prime}, \\
K & =\left[\begin{array}{llll}
-0.4802 & -5.4503 & -14.9865 & -2.9440
\end{array}\right],
\end{aligned}
$$

the closed-loop system remains stable even in the presence of variable measurement channel delay smaller than 0.095 (note that $\tau_{i \max }=h^{a}+\tau_{k}^{s}+\left(t_{k+1}^{s}-t_{k}^{s}\right)$ ). Fig. 4(a), Fig. 4(b) show the norm of $x(t)$ and $e(t)$ respectively when the pendulum's non-linear model in [8] is used with the initial condition $\left[\begin{array}{llll}0.1 & 0.1 & 0 & 0.15\end{array}\right]$.

\section{5 conclusion and future work}

We proposed two types of control units: non-anticipative and anticipative. NCSs with an LTI plant model and anticipative or non-anticipative controller can be modeled by a DDE such as (11). We found sufficient conditions for asymptotic stability of (11) in the form of matrix inequalities and presented a procedure to design output feedback control unit for NCSs. Our method shows significant improvement in comparison to the existing results.

We will extend our results to $H_{\infty}$ or $H_{2}$ design. The problem of stabilizing the plant in the presence of saturation in the control loop will also be considered.

\section{Appendix}

Equation (11) can be written in as equivalent form [5]

$$
\dot{x}(t)=y(t),-y(t)+\sum_{i=0}^{2} A_{i} x(t)-\sum_{i=1}^{2} A_{i} \int_{t-\tau_{i}}^{t} y(s) d s=0,
$$

and the following Lyapunov-Krasovskii functional: 
$V(t)=x^{\prime} P_{1} x+\sum_{i=1}^{2} \int_{-\tau_{i \max }}^{0} \int_{t+\theta}^{t} y^{\prime}(s) R_{i} y(s) d s d \theta+\sum_{i=1}^{2} \int_{t-\tau_{i \min }}^{t} x^{\prime}(s) S_{i} x(s) d s$,

where $P_{1}>0$. Differentiating the first, second and third term of (19) with respect to $t$ respectively gives

$$
\begin{aligned}
& 2 x^{\prime} P_{1} \dot{x}(t)=2 \tilde{x}^{\prime}(t) P^{\prime}\left[\begin{array}{l}
\dot{x} \\
0
\end{array}\right] \\
& \sum_{i=1}^{2}\left(\tau_{i \max } y^{\prime}(t) R_{i} y(t)-\int_{t-\tau_{i \max }}^{t} y^{\prime}(\tau) R_{i} y(\tau) d \tau\right), \\
& \sum_{i=1}^{2}\left(x^{\prime}(t) S_{i} x(t)-x^{\prime}\left(t-\tau_{i \min }\right) S_{i} x\left(t-\tau_{i \min }\right)\right),
\end{aligned}
$$

where $\tilde{x}=\left[x(t)^{\prime} y(t)^{\prime}\right]^{\prime}$. Substituting (18) into (20a),

$$
\begin{aligned}
\frac{d V(t)}{d t} \leq \tilde{x}^{\prime}(t) \tilde{\Psi} \tilde{x}-\sum_{i=1}^{2} x^{\prime}\left(t-\tau_{i \min }\right) S_{i} x\left(t-\tau_{i \min }\right)- \\
\sum_{i=1}^{2} \int_{t-\tau_{i \max }}^{t} y^{\prime}(\tau) R_{i} y(\tau) d \tau+\eta
\end{aligned}
$$

where

$$
\begin{aligned}
& \tilde{\Psi}=P^{\prime}\left[\begin{array}{cc}
0 & I \\
\sum_{i=0}^{2} & A_{i}-I
\end{array}\right]+\left[\begin{array}{cc}
0 & I \\
\sum_{i=0}^{2} & A_{i}-I
\end{array}\right]^{\prime} P+\sum_{i=1}^{2}\left[\begin{array}{cc}
S_{i} & 0 \\
0 & \tau_{i \max } R_{i}
\end{array}\right], \\
& \eta=\sum_{i=1}^{2} 2 \tilde{x}^{\prime}(t) P^{\prime}\left[\begin{array}{c}
0 \\
A_{i}
\end{array}\right] \int_{t-\tau_{i}}^{t} y(s) d s .
\end{aligned}
$$

By Moon-park inequality [12], a bound on cross term, $\eta$, can be found as follows: 


$$
\begin{aligned}
& \eta \leq \sum_{i=1}^{2} \int_{t-\tau_{i \min }}^{t}\left[\begin{array}{l}
y(s) \\
\tilde{x}(t)
\end{array}\right]^{\prime}\left[\begin{array}{cc}
R_{i} & T_{i}-\left[\begin{array}{ll}
0 & A_{i}^{\prime} \\
* & Z_{1 i}
\end{array}\right] P
\end{array}\right]\left[\begin{array}{l}
y(s) \\
\tilde{x}(t)
\end{array}\right] d s \\
& +\sum_{i=1}^{2} \int_{t-\tau_{i}}^{t-\tau_{i \min }}\left[\begin{array}{l}
y(s) \\
\tilde{x}(t)
\end{array}\right]^{\prime}\left[\begin{array}{cc}
R_{i} & \tilde{T}_{i}-\left[\begin{array}{ll}
0 & A_{i}^{\prime}
\end{array}\right] P \\
* & Z_{2 i}
\end{array}\right]\left[\begin{array}{l}
y(s) \\
\tilde{x}(t)
\end{array}\right] d s \\
& =\sum_{i=1}^{2} \int_{t-\tau_{i}}^{t} y^{\prime}(s) R_{i} y(s) d s \\
& +2 \sum_{i=1}^{2} \int_{t-\tau_{i}}^{t-\tau_{i} \min } y^{\prime}(s)\left(\tilde{T}_{i}-\left[\begin{array}{ll}
0 & A_{i}
\end{array}\right] P\right) \tilde{x}(t) d s \\
& +2 \sum_{i=1}^{2} \int_{t-\tau_{i \min }}^{t} y^{\prime}(s)\left(T_{i}-\left[0 A_{i}\right] P\right) \tilde{x}(t) d s \\
& +\sum_{i=1}^{2} \tilde{x}^{\prime}(t)\left(\tau_{i \min } Z_{1 i}+\left(\tau_{i}-\tau_{i \min } Z_{2 i}\right) \tilde{x}(t),\right.
\end{aligned}
$$

where

$$
\left[\begin{array}{cc}
R_{i} & T_{i} \\
* & Z_{1 i}
\end{array}\right] \geq 0, \quad\left[\begin{array}{cc}
R_{i} & \tilde{T}_{3} \\
* & Z_{2 i}
\end{array}\right] \geq 0
$$

By choosing $\tilde{T}_{i}=\left[\begin{array}{ll}0 & A_{i}^{\prime}\end{array}\right] P$,

$$
\begin{aligned}
& \eta \leq \sum_{i=1}^{2}\left(\int_{t-\tau_{i}}^{t} y(s)^{\prime} R_{i} y(s) d s+2 x^{\prime}(t)\left(Y_{i}-\left[0 A_{i}^{\prime}\right] P\right) \tilde{x}(t)\right) \\
& -2 \sum_{i=1}^{2}\left(x^{\prime}\left(t-\tau_{i \min }\right)\left(Y_{i}-\left[0 A_{i}^{\prime}\right] P\right) \tilde{x}(t)\right) \\
& +\sum_{i=1}^{2}\left(\tilde{x}^{\prime}(t)\left(\tau_{i \min } Z_{1 i}+\left(\tau_{i \max }-\tau_{i \min }\right) Z_{2 i}\right) \tilde{x}(t) .\right.
\end{aligned}
$$

Based on the Lyapunov-Krasovskii theorem, (11) is asymptotically stable if $\frac{d V}{d t} \leq-\varepsilon\|x\|^{2}$ for some $\varepsilon>0$. Hence the system is asymptotically stable if (12a) holds. However any row and column of (12a) except the first block row and column can be zero. The inequalities in (12b) and (12c) are in fact nonstrict. However for simplicity and since there is no numerical advantage we state them as strict inequality.

\section{References}

[1] M. S. Branicky, S. M. Phillips, and W. Zhang. Stability of networked control systems:explicit analysis of delay. In Proc. of the 2000 Amer. Contr. Conf., pages 2352-2357, June 2000. 
[2] M. C. de oliveira, J. F. Camino, and R. E. Skelton. A convexifying algorithm for the design of structured linear controllers. In Proc. of the 39th Conf. on Decision and Contr., pages 2781-2786, Dec 2000.

[3] E. Fridman, A. Seuret, and J. P. Richard. Robust sampel-data stabilization of linear systems: an input delay approach. Automatica, 40(8): 1441-1446, Aug. 2004.

[4] E. Fridman and U. Shaked. A descriptor system approach to $H_{\infty}$ control of linear time-delay systems. IEEE Trans.on Automatic Control, 47(2): 253-270, Feb. 2002.

[5] E. Fridman and U. Shaked. An improved stabilization method for linear time-delay systems. IEEE Trans.on Automatic Control, 47(11), Nov. 2002.

[6] L. E. Ghaoui, F. Oustry, and M. AitRami. A cone complementarity linearization algorithm for static output-feedback and related problems. IEEE Trans. on Automat. Contr., 42(8):1171-1176, Aug. 1997.

[7] J. P. Hespanha, P. Naghshtabrizi, and Y. Xu. Networked control systems:analysis and design. Submitted to - Special Issue of the Proceedings of the IEEE on The Emerging Technology of Networked Control Systems.

[8] M. Landry, S. A. Campbell, K. Morris, and C. O. Aguilar. Dynamics of an inverted pendulum with delayed feedback control. SIAM J. on Applied Dynamical Syatems, 4(2):333-351, 2005.

[9] H. Lin, G. Zhai, and P. J. Antsaklis. Robus stability and disturbance attennuation analysis of a class of networked control systems. In Proc. of the 42th Conf. on Decision and Contr., pages 1182-1187, Dec. 2003.

[10] B. Lincoln and B. Bernhardsson. Optimal control over networks with long random delays. In Proc. of the 14th Int. Symp. of Mathematical Th. of Networks and Syst., 2000.

[11] L. A. Montestruque and P. J. Antsaklis. On the model-based control of networked systems. Automatica, 39(10):1837-1843, Oct. 2003.

[12] Y. S. Moon, P. Park, W. Kwon, and Y. Lee. Delay-dependent robust stabilization of uncertain state -delayed systems. Int. Journal of Control, (74):1447-1455, 2001.

[13] P. Naghshtabrizi and J. P. Hespanha. Designing observer-based controller for network control system. To be presented at the IEEE CDC., Dec. 2005.

[14] D. Nesic and A. Teel. Input-output stability properties of networked control systems. IEEE Trans. on Automat. Contr., 49(10):1650-1667, Oct. 2004.

[15] J. Nilsson. Real-Time Control Systems with Delays. PhD thesis, Department of Automatic Control, Lund Institute of Technology, Sweden, Jan. 1998.

[16] G. C. Walsh, H. Ye, and L. Bushnell. Stability analysis of networked control systems. In Proc. of the 1999 Amer. Contr. Conf., pages 28762880, June 1999. 
[17] G. C. Walsh, H. Ye, and L. Bushnell. Stability analysis of networked control systems. IEEE Trans. on Contr. Syst. Tech., 10(3):438-446, May 2001.

[18] M. Yu, L. Wang, T. Chu, and F. Hao. An LMI approach to networked control systems with data packet dropout and transmission delays. $J$. of Hybrid Syst., 3(11), Nov. 2004.

[19] W. Zhang and M. S. Branicky. Stability of networked control systems with time-varying transmission period. In Allerton Conf. of Communication, contr. and computing, Urbana, IL, Oct. 2001. 